\title{
Splenic immunity and atherosclerosis: a glimpse into a novel paradigm?
}

Commentary

See related article, pages $745-753$.

\author{
Joseph L. Witztum \\ Department of Medicine, University of California, San Diego, La Jolla, California 92093-0682, USA \\ Phone: (858) 534-4347; Fax: (858) 534-2005; E-mail: jwitztum@ucsd.edu.
}

J. Clin. Invest. 109:721-724 (2002). DOI:10.1172/JCI200215310.

\begin{abstract}
Atherosclerosis is a chronic disease that involves a complex interaction of the artery wall and its cellular elements, lipoproteins, and circulating blood elements. Although hypercholesterolemia is a prerequisite for the initiation of atherogenesis, once initiated, the atherosclerotic lesion takes on many of the characteristics of a chronic inflammatory process $(1,2)$. In particular, there is now strong and growing evidence in hypercholesterolemic animal models that the immune system is specifically activated by atherosclerosis-associated antigens and that both innate and adaptive immunity can significantly modulate subsequent lesion development.
\end{abstract}

\section{Immune recognition of the atherosclerotic plaque}

$\mathrm{T}$ cells, which mediate adaptive immunity, are an early and important component of lesions, and macrophages, which play a key role in both lesion development and in mediating plaque disruption, serve to bridge innate and adaptive immune processes. Immuno-globulins are abundant in the plaque, in part complexed to lesion epitopes, such as "oxidationspecific" epitopes of oxidized LDL (OxLDL). However, B cells themselves are noticeable for their absence, though they have been reported in the adventitia surrounding diseased vessels. Specific humoral, as well as cellular immunity has been demonstrated to a number of antigens in the atherosclerotic lesion, including viral and bacterial antigens, heat-shock proteins, modified arterial wall elements, and especially OxLDL. Indeed, autoantibodies to a variety of oxidation-specific epitopes of OxLDL, such as that modeled by malondialdehydemodified LDL (MDA-LDL), have generally been shown to correlate with the extent of lesion formation in mice and humans $(3,4)$.
Evidence that immune responses are important in the natural history of lesion progression comes from many studies now, demonstrating that modulation of specific immune components affects both the rate of atherogenesis as well as the composition of the lesion itself. For example, $A p o E^{-/-}$and $L D L R^{-/-}$mice have been crossed into a $\operatorname{Rag}(1 \mathrm{or} 2)^{-/-}$background, generating hypercholesterolemic mice with a deficiency of both $T$ and $B$ cells. If these mice are fed a high fat diet to produce exceptionally high plasma cholesterol levels (>1,300 mg/dl), after a sufficient period of time (16-22 weeks), the extent of atherogenesis is not affected (5-7). However, at earlier time points (4-8 weeks), or even at more extended periods of time in the presence of more moderate cholesterol levels $(600-800$ $\mathrm{mg} / \mathrm{dl}$ ), the combined immunodeficiency does alter the course of atherogenesis, resulting in a $40-80 \%$ decreased rate of lesion formation $(5,7,8)$.

These studies teach us that if the atherogenic pressure is exceedingly high, immune function is not obligatory for the initiation or progression of lesion formation, but under conditions of more moderate atherogenic pressure, the immune responses play a proatherogenic role. Indeed, most studies of immune modulation to date have indicated a proatherogenic influence of the immune system on atherogenesis. For example, neutralizing CD40L interactions, either genetically or by infusion of blocking antibodies, reduces lesion formation (9), as does blocking of the activity of the Th1 cytokine INF- $\gamma(10)$. This subject has been covered in depth in several recent reviews $(4,11,12)$.

During evolution, an increasing number of genes have been devoted to the expansion of what can be collectively called immune function. Surely, the genetic selection of such a complex system must have some important survival advantage. Atherosclerosis is only manifested beyond the reproductive period, and thus is not likely to exert any evolutionary pressure itself. However, it is now apparent that there are generalized responses to inflammatory components of the atherogenic process that are shared with those seen in infectious and acute and chronic diseases. It is likely that such responses exert selective positive pressure on immune components that could also exercise protective mechanisms with regard to atherogenesis. For example, hyper-immunization of hypercholesterolemic rabbit and murine models with OxLDL epitopes, so as to achieve very high plasma titers of antibodies to OxLDL, ameliorates atherogenesis (12), as does the intravenous administration of polyspecific $\operatorname{IgG}$ isolated from pooled donors (13). $A \mathrm{POE}^{-/-}$mice develop very high titers of IgM autoantibodies to a variety of oxidation-specific neoepitopes on OxLDL (14). Furthermore, it has recently been demonstrated that $\operatorname{IgM}$ monoclonal autoantibodies to OxLDL, cloned from the spleens of such cholesterol-fed ApoE $E^{-/-}$mice, specifically bind to oxidized phospholipid epitopes and block the uptake of OxLDL by macrophage scavenger receptors, a key step in the formation of the atherosclerotic plaque. Presumably, such inhibition of OxLDL uptake serves an anti-atherogenic function, as genetic deletion of scavenger receptors protects against atherogenesis in some genetic backgrounds. These antibodies also bind to apoptotic cells, which display similar oxidized phospholipid epitopes. Interestingly, these antibodies, which are secreted by the spleen, have been shown to be identical to the T15 clonospecific anti-phosphorylcholine (anti-PC) antibodies, which arise without prior immunization and do not require germline recombination to achieve specificity for their target epitopes (15-17). These so-called "natural" 
antibodies confer optimal protection to mice against lethal infection with encapsulated organisms, such as Streptococcus pneumoniae. Because these germline antibodies are found by day 7 in mice maintained in germ-free environments, it has been postulated that they are actually positively selected for their ability to clear oxidized membranes found on apoptotic cells. Subsequent exposure to microbial PC serves to exert further positive selection pressure. These antibodies are greatly expanded in the cholesterolfed $A p o E^{-/-}$mice, presumably because of the presence of excess OxLDL (18). Thus, while much evidence supports a pathogenic role for many immune mediated mechanisms, there is, not surprisingly, also precedent for the observation that some aspects of immunity serve to decrease the progression of atherosclerosis as well.

\section{Physiological roles of the spleen}

The spleen plays an important, though not obligatory role, in immune function. In general, patients who have undergone splenectomy do not suffer fundamental defects in immune function, although up to $6 \%$ can experience the "overwhelming postsplenectomy infection syndrome," with particular susceptibility to encapsulated organisms such as S. pneumoniae and Hemophilus influenza. The spleen plays a primary role in blood filtration, such as removal and destruction of aged or damaged erythrocytes or other blood cells, as well as in the removal of other particulate components such as immune complexes, bacteria, or colloidial particles. It has generally been thought that a major reason for the susceptibility of splenectomized patients to specific infections is the loss of this ability to clear the plasma of immune complexes and/or certain encapsulated bacteria like S. pneumoniae.

In addition, the spleen plays an important homeostatic role in immune function by trapping and processing antigens, homing, transforming and proliferating lymphocytes, and activating macrophages. This organ is thought to include up to $25 \%$ of all memory B cells. It is a major site of antibody production, particularly against antigens that are $\mathrm{T}$ cell-independent (TI), such as bacterial capsular polysaccharide, which typifies the rapid immune response to encapsulated bacteria in normal individuals (19). The spleen is the major site of secretion of natural antibodies generated by a subset of B cells, termed B-1 cells. The rapid initial host response to bacteria and viruses is due in part to the constitutive expression and immediate induction of T15 and other such natural antibodies (20). Such TI antigens are typically composed of closely spaced repeated epitopes, such as can be found on a haptenated bead containing an epitope expressed at high density (21). It may be speculated that OxLDL, which contains multiple copies of oxidation-specific neoepitopes on a single $200 \AA$ article, is such an antigen.

\section{Splenectomy accelerates atherogenesis}

Given the general immunocompetence of most splenectomy patients, it is therefore of considerable interest and surprise that Caligiuri and colleagues report in this issue of the JCI that splenectomy dramatically accelerates atherosclerosis in $A p o E^{-/-}$mice fed an atherogenic diet, compared with similar mice subjected to sham operation (22). Mice were maintained in isolator units, treated with antibiotics, fed sterilized food, and gained weight equal to that of controls. This suggests that infection was not the etiology of the accelerated disease.

A previous report also demonstrated that splenectomy in cholesterolfed rabbits led to enhanced lesion formation compared to sham-operated rabbits. However, in that study, total cholesterol and triglyceride levels were higher, and HDL levels were lower in the splenectomized animals in response to the cholesterol diet, and the enhanced atherosclerosis was attributed to the greater degree of hyperlipidemia (23). Splenectomy in rats has also been reported to increase plasma cholesterol and triglycerides and to decrease HDL (24). However, in the present study, feeding a "Western diet" resulted in exceedingly high plasma cholesterol levels $(\sim 1,300 \mathrm{mg} / \mathrm{dl})$ in both splenectomized and sham-operated $A p o E^{-/-}$ mice. Although triglyceride and HDL levels were not reported, in the face of such marked hypercholesterolemia it is unlikely that changes in lipoprotein levels or composition accounted for the aggravation of lesion formation observed. Future studies should address this possibility.
Thrombocytosis is another major problem postsplenectomy in humans and can lead to enhanced thromboembolic disease. Postsurgery platelet levels were not reported for these mice, nor if any long-term increases in morbidity or mortality occurred. Although platelet microparticles have been reported to drive foam-cell formation in vitro (25), there is as yet no evidence that this occurs in vivo. Changes in lipoprotein composition or platelet levels, or loss of filtration capacity of immune complexes, bacteria or other antigens are all unlikely causes of the accelerated atherogenesis in this study, as the enhanced disease in the splenectomized mice was reported to be fully rescued by the passive transfer of immune splenic cells from $A p o E^{-/-}$mice.

\section{Transfer of $T$ cells and $B$ cells rescues the atherogenic effect of splenectomy}

The authors report that the enhanced atherosclerosis following splenectomy could be abrogated by the passive transfer of splenic cells isolated from $A p o E^{-/-}$ mice and that this rescue was more complete if the cells were obtained from donors that were older and therefore had greater disease. The authors argue that these cells were thus more "educated" and were able to transfer enhanced specific immunocompetence that provided protection from disease.

These observations might appear to present an internal paradox: If the donors already possessed such antiatherogenic (presumably immunoprotective) splenic cells, why did they still exhibit such advanced disease? One possible answer is that such immunological education occurs only after disease exposure, at which time it may be too late to protect the donor animal. In contrast, transfer of immunocompetent donor cells to the splenectomized mice provided protection at a much earlier stage in disease progression, and at a time when such an intervention was still effective. By analogy, as atherogenesis progresses in $A p o E^{-/-}$(or $L D L R^{-/-}$) mice, there is a parallel and progressive rise in autoantibodies to epitopes of OxLDL. Even if such antibodies are protective, for example by mediating clearance of modified LDL from plasma, or by inhibiting uptake of OxLDL by macrophages, they may not be of sufficient titer to totally protect against disease progression, although they might 
alter the rate of progression. However, if one immunizes such mice with OxLDL to achieve titers far higher than those reached in the absence of such immunization and at a much earlier stage of their disease, then immunoprotection does occur (reviewed in refs. 4, 12).

Other puzzles remain, however. Why should the transfer of naive splenic cells from C57BL/ 6 mice, which do not have hypercholesterolemia or atherosclerosis, also provide protection to the splenectomized mice? Note that while plasma cholesterol levels were reduced to some extent in these mice, possibly due to apoE production by the transferred cells, they were not substantially lower than in other groups of mice that enjoyed no reduction in atherogenesis. Similarly, it is equally hard to understand why transfer of splenic B-cells to the sham-operated $A p o E^{-/-}$mice, which should have retained the full atheroprotective properties of the spleen, also conferred additional protection.

Confusion also surrounds the issue of which splenic cell types confer the apparent protection from atherosclerosis. The authors report that transfer of purified splenic $\mathrm{T}$ cells (consisting of $60 \% \mathrm{CD}^{+}$and $40 \% \mathrm{CD}^{+}$) isolated from aged $A p o E^{-/}$mice provided a full rescue of the adverse impact of splenectomy. This is surprising in that they had previously reported that transfer of $\mathrm{CD}^{+} \mathrm{T}$ cells from atherosclerotic donors accelerated lesion formation in $A p o E^{-/-} / S C I D$ mice (26). Remarkably, transfer of B cells alone also appears to provide a full rescue. Furthermore, the authors report that the B cell transfer actually conveys a net protective effect on atherogenesis in the splenectomized mice - even beyond that seen with the "full rescue" achieved by the whole spleen cell transfer. Because approximately equal numbers of B cells were transferred in the whole cell preparations (43\% of 60 million cells) as in the purified B cell transfers ( 20 million), it follows that the B cell transfer alone was more protective than the whole cell transfer. If this unusual finding holds up on further study, it may be that interactions among the various transferred cell populations affect the efficiency of the rescue. Alternatively, some subpopulation of T cells, possibly the $\mathrm{CD}^{+}$cells as noted above, might have an adverse effect on this process. In fact, the authors reported that such a B cell transfer to sham-operated mice was also protective, whereas transfer of $\mathrm{T}$ cells was not, and was even marginally adverse. One wonders whether B cells (or T cells) harvested from other organs would confer equal protection. In other experimental models of immune mediated disease, transferred lymphocytes obtained from the spleen or from lymph nodes of the same donor had differential outcomes on the disease process in the recipient (27).

\section{Possible effects of splenic cells on disease progression}

The transfer of splenic cells might affect the progression of atherogenesis through at least two general mechanisms. First, it might lead to the direct homing of immune cells to the lesion, where they might exert direct local effects. Considerable evidence suggests that $\mathrm{T}$ cells in the developing atherosclerotic lesion directly affect lesion size and composition through multiple mechanisms $(4,11)$. In order to demonstrate engraftment and maturation of the transferred T cells, Caligiuri et al. isolated $T$ cells from mice transgenic for the thymidine kinase $(T K)$ gene under control of the CD4 promoter, which results in TK expression in mature T cells (22). Analysis of tissue mRNA of mice 4 weeks after transfer of such cells revealed a relative enrichment in lung and thymus, as well as in the spleen of sham-operated mice. Of importance, expression was also detected in lymph nodes and aorta, particularly in splenectomized mice. Thus, some residual engraftment of these $\mathrm{T}$ cells remained in immune organs and aorta after 4 weeks, presumably due to antigen exposure. However, it is unknown if this engraftment persisted for the full 12 week period at which time the extent of atherosclerosis was assessed. Moreover, information about the engraftment of the transferred $B$ cells is not yet available. Because few B cells have ever been reported in lesions, such engraftment would presumably occur elsewhere.

A second general mechanism by which such transfer could affect lesion formation is through mediating regulatory functions on other immune cells and particularly by mediating relevant antibody generation. The authors examined the impact of splenectomy and cell transfer on antibody titers to an epitope of OxLDL, MDA-LDL. The absolute titers of autoantibodies in the untouched $a p o E^{-/-}$mice appear low compared to those previously reported (14), although the methodologies used were different, and it is unclear at what time in relation to the splenectomy or cell transfers that plasma was obtained for the antibody determinations in the present study. Consistent with the fact that the spleen is a significant site of antibody synthesis and a repository of memory B cells, Caligiuri et al. report a reduction in $\operatorname{IgG}$ titers to MDA-LDL in splenectomized animals (22). Transfer of B cells to splenectomized mice produced the greatest rise in IgG titers, while $T$ cell transfers induced no change at all. Interestingly, the rise in anti-MDA-LDL titers was actually lower in the whole cell transfers than in the $B$ cell transfer, possibly suggesting again some regulatory interaction among the transferred cells. Additional studies will be needed to determine the effects on antibody levels following B cell transfer into the sham-operated mice.

With respect to IgM titers to MDALDL, it is surprising that splenectomy caused no decrease, whereas B cell transfer produced only a modest increase and $T$ cell transfer had no impact. As noted above, there is an expansion in the spleens of $A p o E^{-/-}$mice of B-1 cells that secrete T15 clonospecific IgM natural antibodies to OxLDL with the capacity to block the uptake of OxLDL. Such TI antibodies, which bind to oxidized phospholipid epitopes, would not be measured by the use of MDA-LDL as a model antigen. However, it is possible that such antibodies, or others of a similar nature that are specific for other relevant epitopes, could account in part for some of the protective effects observed with splenic immunity.

\section{Concluding thoughts}

As so often happens in science, we are presented with a novel and provocative set of observations, without any clear insight into the mechanisms involved. In cholesterol-fed $A p o E^{-/-}$mice, splenectomy seems to accelerate atherogenesis, implying a protective effect of the spleen. While at first one might have invoked more traditional roles of splenic function in this property, such as antigen or immune complex trapping, or removal of some circulating cellular elements, the fact that transfer of either splenic $\mathrm{T}$ or B cells can rescue the adverse consequences fully suggests this is not correct. It is 
hard to put into standard paradigms how transfer of these elements from young or atherosclerotic $A p o E^{-/-}$ donors could account for the protective effects, especially the reported ability of $B$ cells to exert a net protective benefit even in sham-operated mice, while at the same time not conferring the same benefits to the donors. The fact that both $T$ cells and $B$ cells could each provide a full rescue suggests that the mechanisms involved must be complex. It will be important for these studies to be confirmed in other contexts, including the use of other murine models. Precisely because an understanding of these effects of splenectomy on atherogenesis are unknown and elusive at present, the elucidation of the mechanisms involved will likely be revealing of unexpected ways in which immune function alters the atherogenic process.

These effects of splenic immunity may not only apply to murine models. There are a number of reports documenting a rise in plasma cholesterol and triglyceride levels in patients who undergo splenectomy for various disease entities, such as mylodysplastic disorders (28). However, in those cases, the plasma lipid levels are frequently depressed prior to splenectomy. In individuals undergoing splenectomy for reasons of trauma, the impact on lipid levels appears to be minimal. In 1977, Robinette and Fraumeni (29) reported a long-term follow-up study of 740 American servicemen who had undergone splenectomy because of trauma during the war years of 1939-1945 and compared them to men with nasopharyngitis matched for age, race, and site of hospital admission (overseas or USA). Cause-specific mortality was determined in over $90 \%$ of the men during 1946-1974. The relative risk for dying of ischemic heart disease was increased nearly two-fold, and of 41 deaths, 30 were attributed to acute myocardial infarction and 11 to chronic ischemic heart disease. There was no excess mortality from thromboembolism. Thus, the novel observations described in the paper by Caligiuri and colleagues may have considerable relevance for understanding the role of immunological function in atherogenesis in humans. Further study is warranted.

1. Ross, R. 1999. Atherosclerosis-an inflammatory disease. N. Eng. J. Med. 340:115-126.

2. Glass, C.K., and Witztum, J.L. 2001. Atherosclerosis. The road ahead. Cell. 104:503-516.

3. Palinski, W., and Witztum, J.L. 2000. Immune responses to oxidative neoepitopes on LDL and phospholipids modulate the development of atherosclerosis. J. Intern. Med. 247:371-380.

4. Hansson, G.K. 2001. Immune mechanisms in atherosclerosis. Arterioscler. Thromb. Vasc. Biol. 21:1876-1890.

5. Dansky, H.M., Charlton, S.A., Harper, M.M., and Smith, J.D. 1997. T and B lymphocytes play minor role in atherosclerotic plaque formation in the apolipoprotein E-deficient mouse. Proc. Natl. Acad. Sci. USA. 94:4642-4646.

6. Daugherty, A., et al. 1997. The effects of total lymphocyte deficiency on the extent of atherosclerosis in apolipoprotein E-/- mice. J. Clin. Invest. 100:1575-1580

7. Song, L., Leung, C., and Schindler, C. 2001. Lymphocytes are important in early atherosclerosis. J. Clin. Invest. 108:251-259. DOI:10.1172/JCI200111380.

8. Reardon, C.A., et al. 2001. Effect of immune deficiency on lipoproteins and atherosclerosis in male apolipoprotein E-deficient mice. Arterioscler. Thromb. Vasc. Biol. 21:1011-1016.

9. Schonbeck, U., and Libby, P. 2001. CD40 signaling and plaque instability. Circ. Res. 89:1092-1103.

10. Gupta, S., et al. 1997. IFN-gamma potentiates atherosclerosis in ApoE knock-out mice. J. Clin. Invest. 99:2752-2761.

11. Libby, P., Hansson, G.K., and Pober, J.S. 1999. Atherogenesis and inflamation. In Molecular basis of cardiovascular disease. K.R. Chien, editor. W.B. Saunders Co. Philadelphia, Pennsylvania, USA 349-366.

12. Hörkkö, S., et al. 2000. Immunological responses to oxidized LDL. Free. Radic. Biol. Med. 28:1771-1779.

13. Nicoletti, A., et al. 1998. Immunoglobulin treatment reduces atherosclerosis in apo E knockout mice. J. Clin. Invest. 102:910-918.

14. Palinski, W., et al. 1996. Cloning of monoclonal autoantibodies to epitopes of oxidized lipoproteins from apolipoprotein E-deficient mice. Demonstration of epitopes of oxidized low den- sity lipoprotein in human plasma. J. Clin. Invest. 98:800-814.

15. Hörkkö, S., et al. 1999. Monoclonal autoantibodies specific for oxidized phospholipids or oxidized phospholipid-protein adducts inhibit macrophage uptake of oxidized low-density lipoproteins. J. Clin. Invest. 103:117-128.

16. Chang, M., et al. 1999. Monoclonal antibodies against oxidized low-density lipoprotein bind to apoptotic cells and inhibit their phagocytosis by elicited macrophages: evidence that oxidationspecific epitopes mediate macrophage recognition. Proc. Natl. Acad. Sci. USA. 96:6353-6358.

17. Shaw, P.X., et al. 2000. Natural antibodies with the T15 idiotype may act in atherosclerosis, apoptotic clearance, and protective immunity. J. Clin. Invest. 105:1731-1740.

18. Silverman, G.J., et al. 2000. Neo-self antigens and the expansion of B-1 cells: lessons from atherosclerosis-prone mice. Curr. Top. Microbiol. Immunol. 252:189-200.

19. Ochsenbein, A.F., and Zinkernagel, R.M. 2000 Natural antibodies and complement link innate and acquired immunity. Immunol. Today. 21:624-630.

20. Bendelac, A., Bonora, E., and Kearney, J.F. 2001. Autoreactivity by design: innate B and T lymphocytes. Nat. Rev. 1:177-186.

21. Ochsenbein, A.F., et al. 2000. Correlation of T cell independence of antibody responses with antigen dose reaching secondary lymphoid organs: implications for splenectomized patients and vaccine design. J. Immunol. 164:6296-6302.

22. Caligiuri, G., Nicoletti, A., Poirier, B., and Hansson, G.K. 2002. Protective immunity against ath erosclerosis carried by B cells of hypercholesterolemic mice. J. Clin. Invest. 109:745-753. DOI:10.1172/JCI200207272.

23. Asai, K., Kuzuya, M., Naito, M., Funaki, C., and Kuzuya, F. 1988. Effects of splenectomy on serum lipids and experimental atherosclerosis. Angiology. 39:497-504.

24. Fatouros, M., et al. 1995. Role of the spleen in lipid metabolism. Br. J. Surg. 82:1675-1677.

25. Curtiss, L.K., Black, A.S., Takagi, Y., and Plow, E.F. 1987. New mechanism for foam cell generation in atherosclerotic lesions. J. Clin. Invest. 80:367-373.

26. Zhou, X., Nicoletti, A., Elhage, R., and Hansson, G.K. 2000. Transfer of CD4(+) T cells aggravates atherosclerosis in immunodeficient apolipoprotein E knockout mice. Circulation. 102:2919-2922.

27. Yang, J., et al. 2000. Splenectomy and adoptive cell transfer reveal a prominent role for splenic memory lymphocytes in the development of chronic relapsing experimental autoimmune encephalomyelitis. Scand. J. Immunol. 52:356-361.

28. Aviram, M., Brook, J.G., Tatarsky, I., Levy, Y., and Carter, A. 1986. Increased low-density lipoprotein levels after splenectomy: a role for the spleen in cholesterol metabolism in myeloproliferative disorders. Am. J. Med. Sci. 291:25-28.

29. Robinette, C.D., and Fraumeni, J.F., Jr. 1977 Splenectomy and subsequent mortality in veterans of the 1939-45 war. Lancet. 2:127-129. 